\title{
Erratum
}

\section{Role of SSB Protein in RecA Promoted Branch Migration Reactions}

Stephen C. West, Era Cassuto, and Paul Howard-Flanders

\section{Mol Gen Genet (1982) 186:333-338}

In this paper the $\mu \mathrm{M}$ concentrations of SSB protein were mis-stated. All $\mu \mathrm{M}$ concentrations stated are ten-fold lower than actually used in the experiments. For example, " $0.25 \mu \mathrm{M}$ SSB" should read " $2.5 \mu \mathrm{M}$ SSB".

Concentrations of SSB expressed in pmol are correct as stated. 